\title{
Adaptive wavefront control with asynchronous stochastic parallel gradient descent clusters
}

\author{
Mikhail A. Vorontsov \\ Computational and Information Sciences Directorate, Intelligent Optics Laboratory, Army Research Laboratory, \\ Adelphi, Maryland 20783, and Institute for Systems Research, Intelligent Optics Laboratory, University of Maryland, \\ College Park, 2107 Engineering Research Building, College Park, Maryland 20740 \\ Gary W. Carhart \\ Computational and Information Sciences Directorate, Intelligent Optics Laboratory, Army Research Laboratory, \\ Adelphi, Maryland 20783
}

Received February 27, 2006; accepted April 24, 2006; posted May 17, 2006 (Doc. ID 68316)

A scalable adaptive optics (AO) control system architecture composed of asynchronous control clusters based on the stochastic parallel gradient descent (SPGD) optimization technique is discussed. It is shown that subdivision of the control channels into asynchronous SPGD clusters improves the AO system performance by better utilizing individual and/or group characteristics of adaptive system components. Results of numerical simulations are presented for two different adaptive receiver systems based on asynchronous SPGD clustersone with a single deformable mirror with Zernike response functions and a second with tip-tilt and segmented wavefront correctors. We also discuss adaptive wavefront control based on asynchronous parallel optimization of several local performance metrics-a control architecture referred to as distributed adaptive optics (DAO). Analysis of the DAO system architecture demonstrated the potential for significant increase of the adaptation process convergence rate that occurs due to partial decoupling of the system control clusters optimizing individual performance metrics. (C) 2006 Optical Society of America

OCIS codes: $010.1080,010.3310$.

\section{INTRODUCTION}

Adaptive optics (AO) systems often comprise several wavefront corrector types (tracking and beam steering mirrors, several deformable mirrors, and/or liquid crystal phase modulators) that have quite different dynamical (time-response) characteristics. ${ }^{1-3}$ This "nonuniformity" of wavefront corrector time responses significantly complicates control system architecture and commonly results in a simplified approach being taken where the control loops for all wavefront correctors are "synchronized" based on the temporal dynamics of the wavefront corrector with the slowest time response- "the slowest sets the pace." This artificially induced slowdown of AO system operation may have a significant impact on an adaptive system's capability for compensation of fast-changing atmospheric-turbulence-induced phase aberrations in various AO applications. A desired solution of this problem would be development of adaptive optics architectures composed of asynchronously operating wavefront control subsystems (AO clusters). The need for asynchronous parallel wavefront control in the form of AO clusters also emerges in such applications as adaptive free-space laser communication and relay-mirror-based laser beam projection systems. In both of these system types, adaptive wavefront distortion compensation is performed in remotely located $\mathrm{AO}$ subsystems. In these wavefront control configurations the remotely located AO subsystems are quite difficult to synchronize without data exchange (e.g., using additional optical or rf communication channels ${ }^{4}$ ). On the other hand, the absence of synchroni- zation between remotely located wavefront control systems may result in undesired cross talk between their control loops and compensation process instability.

One more example of the technical challenges faced by conventional AO control techniques is feedback control of super-high-resolution AO mirror arrays consisting hundreds or even thousands of actuators-the so-called extreme adaptive optics. ${ }^{5}$ With the conventional phaseconjugation-based wavefront control approach, a key bottleneck for these extreme-AO systems is the time delay between the wavefront sensor's data measurements and the completion of control signals computation. Typically, the time required for the wavefront sensor data processing increases proportionally to the square of the number of wavefront corrector (AO mirror) actuators. ${ }^{6}$ For this reason the high-resolution phase-conjugation-type AO technique faces serious problems in providing the operational speed required for most atmospheric AO applications.

Compensation operational speed can be an even more serious problem in the alternative to phase-conjugationtype systems based on optimization of a system performance metric (e.g., Strehl ratio, ${ }^{1}$ sharpness functions, ${ }^{7}$ or image quality metrics ${ }^{8}$ ). The metric optimization is commonly performed using iterative wavefront control algorithms such as gradient descent, multidithering, and stochastic parallel gradient descent (SPGD). ${ }^{1,8,9}$ With these wavefront control techniques the iterative process convergence speed-on which the AO system compensation bandwidth depends-rapidly decreases with increasing 
control channel number or, equivalently, AO compensation spatial resolution. From this viewpoint, subdivision of the AO system's control channels into parallel weakly coupled control clusters, each with a smaller number of control channels, may significantly improve convergence speed and the AO system's bandwidth. ${ }^{10}$ The AO wavefront control architectures described here are based on the use of asynchronous SPGD AO clusters. It is shown that wavefront control based on asynchronous SPGD AO clusters represents an attractive alternative to traditional $\mathrm{AO}$ wavefront control approaches for various adaptive optics applications.

\section{STOCHASTIC PARALLEL GRADIENT DESCENT CONTROL CLUSTERS: PROBLEM STATEMENT}

The notional schematic of an AO receiver system with control clusters is shown in Fig. 1. In this system the control channels are distributed between $N_{c}$ asynchronously operating control clusters with $M_{j},\left(j=1, \ldots, N_{c}\right)$ channels in each cluster. There is an advantage (but not a requirement) to assign AO system control channels to individual clusters based on some common characteristic such as, for example, wavefront corrector actuator time responses as mentioned, actuator physical locations (e.g., control chan-

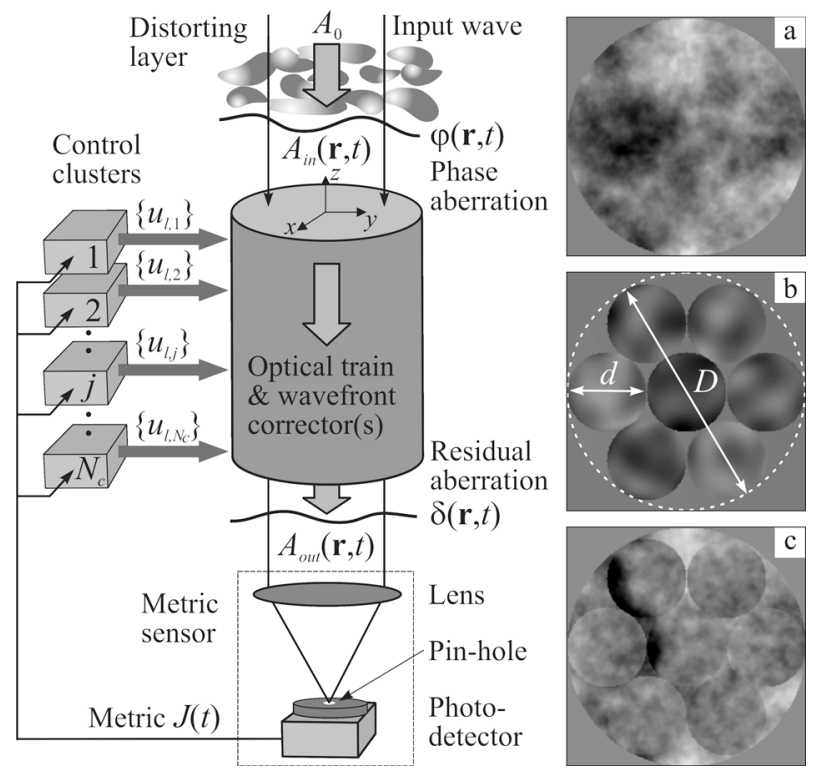

Fig. 1. Schematic of an AO receiver system with wavefront control channels distributed between AO control clusters. Propagation of optical wave $A_{0}$ through a distorting layer results in phase aberration $\varphi(\mathbf{r}, t)$ of received input wave $A_{\text {in }}(\mathbf{r}, \mathrm{t})$ that enters the optical receiver system (shown by a cylinder). Here $\mathbf{r}=\{x, y\}$ is a coordinate vector orthogonal to the AO system's optical axis and $t$ is the time. The receiver system optical train contains one or more wavefront correctors (not shown). The receiver output wave $A_{\text {out }}(\mathbf{r}, \mathrm{t})$ with phase $\delta(\mathbf{r}, \mathrm{t})$ (residual phase) enters a system performance metric sensor sensitive to the residual phase aberration. The metric signal $J$ is sent to controllers (control clusters) that compute voltages $\left\{u_{l, j}\right\}$ that are applied to the wavefront corrector's electrodes. (a) Gray-scale images at right are examples of the pupil plane phase aberration, (b) the phase correction at each subaperture of the segmented wavefront corrector (described in the text) having seven subapertures, and (c) the residual phase distortion. nels belonging to different wavefront correctors can be assigned to separate clusters) or spatial resolution of phase distortion compensation (so that Zernike aberrations of different orders are compensated using different control clusters). A group of control channels associated with a high-resolution wavefront corrector [AO mirror or liquid crystal phase spatial light modulator (SLM)] segment can be a cluster as well as a group of control channels associated with adaptive subapertures of a tiled optical system. $^{11}$

Assume that wavefront distortion compensation in an AO system composed of $N_{c}$ clusters is based on optimization of a measured performance metric $J$. In the AO system schematic shown in Fig. 1, the performance metric is proportional to received light power inside a small pinhole located at the coordinate origin of the lens focal plane (Strehl metric). The metric value $J$ depends on the control voltages $\left\{u_{l, j}\right\}$ applied to AO system actuators, where indices $l\left(l=1, \ldots, M_{j}\right)$ and $j\left(j=1, \ldots, N_{c}\right)$ are associated with the control channel and cluster numbers, correspondingly. Thus the performance metric $J$ is a function of $N_{\Sigma}$ $=\sum_{j=1}^{N_{c}} M_{j}$ control variables.

Consider first an AO control system architecture based on performance metric optimization using the conventional SPGD algorithm. ${ }^{8,12,13}$ SPGD wavefront control uses cyclic (iterative) updates of the control voltages $\left\{u_{l, j}\right\}$ computed based on measurements of the performance metric perturbations $\delta J^{(n)}$, where index $n=0,1, \ldots$, corresponds to the adaptation (optimization) process iteration number. The metric perturbation $\delta J^{(n)}$ results from small amplitude random control voltage changes (perturbations) $\left\{\delta u_{l, j}\right\}$ applied simultaneously to all AO system wavefront corrector electrodes. In the original SPGD approach, control voltage perturbations $\left\{\delta u_{l, j}\right\}$ are assumed to be delta-correlated, random values with zero mean., ${ }^{8,13}$ In more recent "SPGD clones," the perturbations $\left\{\delta u_{l, j}\right\}$ can be statistically dependent (correlated) random variables, so that the wavefront phase perturbations $\delta u(\mathbf{r})$ originating from the control voltage perturbations $\left\{\delta u_{l, j}\right\}$ are statistically correlated with phase aberrations the $\mathrm{AO}$ system intends to compensate. ${ }^{3,12}$ The SPGD control voltage update rule is given by the expression

$$
u_{l, j}^{(n+1)}=u_{l, j}^{(n)}+\gamma_{j}^{(n)} \delta J^{(n)} \delta u_{l, j}^{(n)}
$$

where $\left\{\gamma_{j}^{(n)}\right\}>0$ are update coefficients (gain coefficients).

In the conventional SPGD control algorithm, all the perturbations $\left\{\delta u_{l, j}^{(n)}\right\}$ in Eq. (1) are applied simultaneously and the corresponding metric perturbation $\delta J^{(n)}$ is measured after the delay time, $\tau \geqslant \max _{l, j}\left\{\tau_{l, j}\right\}$ where $\left\{\tau_{l, j}\right\}$ are time responses of the individual channels. This "the slowest sets the pace" time-delay rule is used to guarantee that all transition processes in wavefront corrector actuators and control circuits caused by the applied perturbations or control voltage updates do not impact metric measurements. This means that the SPGD iteration (clock) rate is determined by the time response of the slowest control channel. This requirement poses a problem when using the SPGD wavefront control approach in AO systems having multiple wavefront correctors with different operational bandwidths. 
In the case of sequential addressing of individual actuators (elements) of a high-resolution AO mirror array or liquid crystal phase SLM, the time delay in metric measurements is determined by the corrector frame update time $\tau_{f}$-the time required to address all wavefront corrector elements-but not the time response $\tau_{\mathrm{ac}}$ of individual actuators that can be significantly shorter than $\tau_{f}$. Thus, the SPGD control technique, as well as any other metric optimization technique used for adaptive wavefront control, follows the most conservative operational times management approach: the control voltage update rate is chosen based on the slowest temporal processes occurring in the system- "the slowest sets the pace." Dividing the control channels into parallel asynchronously operating control clusters as discussed in Section 3 intends to improve AO system performance by better utilizing individual and/or group characteristics of adaptive system elements.

\section{DYNAMICAL MODEL OF ASYNCHRONOUS STOCHASTIC PARALLEL GRADIENT DESCENT CLUSTERS}

Assume that control loops (actuators and/or control circuits) belonging to the $j$ th cluster have near the same time response $\tau_{j}$, where $j=1, \ldots, N_{c}$. Describe dynamics of the controlling elements in the $j$ th cluster by the following first-order dynamical model:

$$
\tau_{j} \frac{\mathrm{d} \mathbf{v}_{j}(t)}{\mathrm{d} t}+\mathbf{v}_{j}(t)=\mathbf{u}_{j}(t), \quad j=1, \ldots, N_{c},
$$

where $\mathbf{u}_{j}(t)=\left\{u_{l, j}(t)\right\},\left(l=1, \ldots, M_{j}, j=1, \ldots, N_{c}\right)$ is the vector of control voltages (controls) applied to wavefront corrector actuators in the $j$ th cluster and $\mathbf{v}_{j}(t)=\left\{\nu_{l, j}(t)\right\}$ is the vector of phase modulation amplitudes resulting from these controls.

The wavefront phase $u(\mathbf{r}, t)$ generated in response to applied controls can be represented as

$$
u(\mathbf{r}, t)=\sum_{j=1}^{N_{c}} \mathbf{v}_{j}(t) \mathbf{S}_{j}(\mathbf{r}),
$$

where $\mathbf{S}_{j}(\mathbf{r})=\left\{S_{l, j}(\mathbf{r})\right\}$ and $S_{l, j}(\mathbf{r})$ are the actuators' response functions.

Dynamics of the sensor used for the performance metric $J(t)$ measurements (metric-sensor) can be described by the equation

$$
\tau_{J} \frac{\mathrm{d} J(t)}{\mathrm{d} t}+J(t)=I(t),
$$

where $\tau_{J}$ is the sensor's integration time and $I(t)$ is the light intensity entering the sensor. For example, for the metric-sensor composed of a lens with a small pinhole located at its focus as shown in Fig. 1, the intensity $I(t)$ is proportional to the Strehl ratio $\mathrm{St}(t)$ - a widely used performance metric in adaptive optics.

Consider the SPGD control voltage update rule [Eq. (1)] independently (asynchronously) applied to individual clusters

$$
\mathbf{u}\left(t_{n+1, j}\right)=\mathbf{u}\left(t_{n, j}\right)+\gamma_{j}^{(n)} \delta J_{j}^{(n)} \delta \mathbf{u}\left(t_{n+1, j}\right), \quad j=1, \ldots, N_{c},
$$

where $t_{n, j}$ are the moments in time when control vector updates occur, and $\delta \mathbf{u}\left(t_{n, j}\right)$ are the control vector perturbations for the $j$ th cluster at the $n$th iteration. Assume that control vector updates at different clusters occur asynchronously, that is, time differences $\Delta_{n, j}=t_{n, j+1}-t_{n, j}$ between updates in different clusters are changing over the time (or over the iteration number $n$ ): $\Delta_{n, j}=\Delta_{j}(n)$. The time differences $\left\{\Delta_{j}(n=0)\right\}$ are referred to here as the initial time offset parameters.

The time durations $\left\{T_{j}\right\}$ between subsequent control voltage updates performed by each cluster control loop depend on the corresponding time responses $\left\{\tau_{j}\right\}$ in Eq. (2). Ideally the times $\left\{T_{j}\right\}$ should be significantly longer than the clusters' response times $\left\{\tau_{j}\right\}\left(T_{j} \gg \tau_{j}\right)$ to ensure that all control loop transition processes are ended prior to the next control voltage updates. On the other hand, the update times $\left\{T_{j}\right\}$ define the clusters' operational speeds (iteration rates) and from this viewpoint it is desirable to make $\left\{T_{j}\right\}$ short (but still without causing AO compensation performance degradation). It is convenient to link the update and response times for all clusters by the following expressions $T_{j}=\beta_{T} \tau_{j}$, where $\beta_{T} \geqslant 1$ is a feedback delay factor.

The control cycle time diagram for the SPGD cluster operation is shown in Fig. 2 for two clusters. In the $j$ th cluster it begins at moment $t_{n, j}$, corresponding to the control vector $\mathbf{u}\left(t_{n, j}\right)$ update as shown in Fig. 2(a). Performance metric measurement $J\left(t_{n, j}+T_{j}\right)$ is performed after the time delay $T_{j}$. Assume that control perturbations $\delta \mathbf{u}\left(t_{n, j}\right)$ are applied to the cluster's actuators at moment $t_{n, j}+T_{j}$ simultaneously with the metric measurement. Measurement of the perturbed metric value $J\left(t_{n, j}+2 T_{j}\right)$ as well as the computation of the metric perturbation $\delta J_{j}^{(n)}$ $=J\left(t_{n+1, j}\right)-J\left(t_{n, j}+T_{j}\right)$ occurs at moment $t_{n+1, j}=t_{n, j}+2 T_{j}$, that is, after delay $T_{j}$ in respect to applied control voltage perturbation. The next control voltages update occurs at the time $t_{n+1, j}+\delta t$, where $\delta t$ is a small time delay required for computations of the new controls. In most cases the computation time $\delta t$ can be ignored $\left(\delta t \ll T_{j}\right)$. Thus the control cycle (iteration) duration for the $j$ th cluster equals to $2 T_{j}$.

The SPGD control loops for different clusters are coupled through the metric values $J(t)$ that depend on control voltage changes occurring in all clusters. Thus, owing to the use of a metric common for all channels, the clusters are coupled.

\section{ANALYSIS}

The key question to answer is, "Why should the asynchronously operated network of SPGD AO clusters as described above provide performance metric optimization?" To address this question, consider metric perturbation $\delta J_{j}^{(n)}=J\left(t_{n, j}+2 T_{j}\right)-J\left(t_{n, j}+T_{j}\right)$ resulting from the applied perturbations and control voltage updates that occurred in all clusters during the time interval $\left(t_{n, j}+T_{j}, t_{n, j}+2 T_{j}\right)$. The metric perturbation $\delta J_{j}^{(n)}$ is computed at $t=t_{n+1, j}$ and used directly for the control voltages update only in the $j$ th cluster. Note that the metric perturbation value $\delta J_{j}^{(n)}$ 


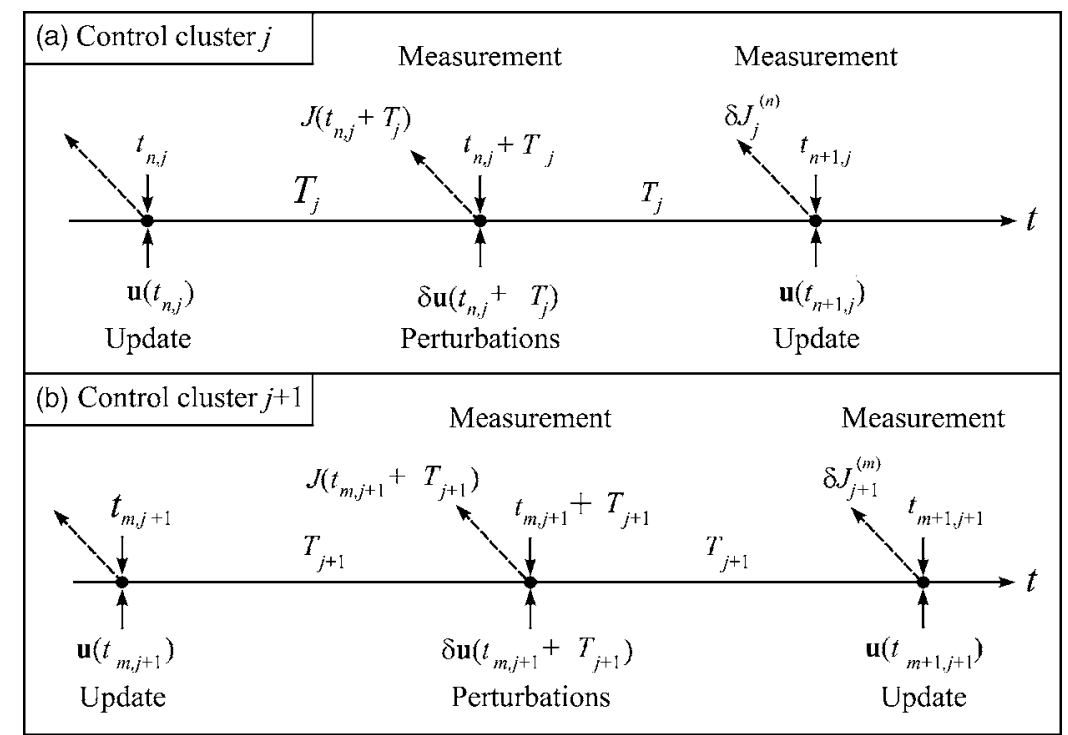

Fig. 2. Operational time diagrams for two asynchronous SPGD clusters. Arrows indicate the moments of metric measurements (dashed lines) and the control voltage updates and perturbations (solid lines).

is influenced by all control voltages changes occurring between moments $t_{n, j}+T_{j}$ and $t_{n, j}+2 T_{j}$ including not only the control voltage perturbations $\delta \mathbf{u}\left(t_{n, j}\right)$ performed by the $j$ th cluster, but also the perturbations $\delta \mathbf{u}\left(t_{m, q}\right)$ and control voltage updates $\Delta \mathbf{u}\left(t_{m^{\prime}, q^{\prime}}\right)$ corresponding to all other clusters for which time moments $t_{m, q}$ and $t_{m^{\prime}, q^{\prime}}$ belong to the interval $\left(t_{n, j}+T_{j}, t_{n, j}+2 T_{j}\right)$. The contributions to metric perturbation $\delta J_{j}^{(n)}$ originating from these other clusters characterize the intercluster cross talk. Despite this intercluster cross talk, the applied perturbation $\delta \mathbf{u}\left(t_{n, j}+T_{j}\right)$ and the metric perturbation measurement $\delta J_{j}^{(n)}$ at $t_{n, j}+2 T_{j}$ are always separated by the "correct" (in respect to the actuators dynamics of $j$ th cluster) time interval $T_{j}$ for only the $j$ th cluster. All other influences on metric perturbations coming from other clusters that are not synchronized with the $j$ th cluster are random and occur at random moments inside the interval $\left(t_{n, j}+T_{j}, t_{n, j}\right.$ $+2 T_{j}$ ). On average, the influence of the intercluster cross talk appears to be significantly smaller than the synchronized in time perturbations and metric measurements of the $j$ th cluster. This qualitative explanation is supported by the following mathematical arguments.

Consider the control voltage update $\Delta u_{l, j}^{(n)}=u_{l}\left(t_{n+1, j}\right)$ $-u_{l}\left(t_{n, j}\right)$ corresponding to the $l$ th control channel in the $j$ th cluster. In accordance with the SPGD update rule [Eq. (5)], $\Delta u_{l, j}^{(n)}=\gamma_{j}^{(n)} \delta J_{j}^{(n)} \delta u_{l, j}^{(n)}$, where $\delta u_{l, j}^{(n)} \equiv \delta u_{l}\left(t_{n, j}\right)$. Using a Taylor series expansion of $\delta J_{j}^{(n)}$ at the moment $t_{n, j}+T_{j}$, represent the product $\delta J_{j}^{(n)} \delta u_{l, j}^{(n)}$ in the form

$$
\begin{aligned}
\delta J_{j} \delta u_{l, j} \cong & \frac{\partial J_{j}}{\partial u_{l, j}}\left(\partial u_{l, j}\right)^{2}+\sum_{k \neq l}^{M_{j}} \frac{\partial J_{j}}{\partial u_{k, j}} \delta J_{k, j} \delta u_{l, j} \\
& +\sum_{q \neq j}^{N_{c}} \sum_{k=1}^{M_{k}} \frac{\partial J_{j}}{\partial u_{k, q}} \delta J_{k, q} \delta u_{l, j} \\
& +\sum_{q^{\prime} \neq j}^{N_{c}} \sum_{k^{\prime}=1}^{M_{k^{\prime}}} \frac{\partial J_{j}}{\partial u_{k^{\prime}, q^{\prime}}} \Delta u_{k^{\prime}, q^{\prime}} \delta u_{l, j} .
\end{aligned}
$$

To simplify notation, we omitted in approximation (6) the iteration index $n$. In the Taylor series expansion [approximation (6)], we keep only the first order terms in respect to the control voltage perturbations and updates terms, assuming that both are small. The first two terms in approximation (6) describes impact on metric perturbation originated from perturbations of control voltages at the $j$ th cluster. The other two terms are due to the intercluster cross talk caused by perturbations and control voltage updates occurred during the time interval $\left(t_{n, j}+T_{j}, t_{n, j}\right.$ $\left.+2 T_{j}\right)$. Because the clusters are not synchronized the double sums in approximation (6) include only contributions from the control voltage perturbations and updates corresponding to the time moments $t_{k, q}$ and $t_{k^{\prime}, q^{\prime}}$ belonging to the interval $\left(t_{n, j}+T_{j}, t_{n, j}+2 T_{j}\right)$. Thus the sums do not include contributions from all control channels.

If the chosen perturbations are random and statistically independent, the second and the third terms in approximation (6) reduce to zero in expected values. Assuming that the perturbations $\left\{\delta u_{l, j}\right\}$ in $j$ th cluster and the updates $\left\{\Delta u_{k^{\prime}, q^{\prime}}\right\} \quad\left(q^{\prime} \neq j\right)$ occurring in other clusters at time interval $\left(t_{n, j}+T_{j}, t_{n, j}+2 T_{j}\right)$ are statistically independent, the expected value of the last term in approximation (6) also can be neglected as the sum of statistically independent terms. Thus, the approximation (6) reduces in the expected value to $\delta J_{j} \delta u_{l, j} \cong\left(\partial J_{j} / \partial u_{l, j}\right)\left(\partial u_{l, j}\right)^{2}$. The expected value of the SPGD update term in Eq. (5) then can be represented in the form

$$
\Delta u_{l, j}^{(n)}=\gamma_{j}^{(n)} \delta J_{j}^{(n)} \delta u_{l, j}^{(n)} \cong \gamma_{j}^{(n)} \frac{\partial J_{j}^{(n)}}{\partial u_{l, j}^{(n)}}\left(\partial u_{l, j}^{(n)}\right)^{2}, \quad j=1, \ldots, N_{c}
$$

This means that the control voltage update is proportional (on average) to the metric $J$ true gradient component in respect to the control variable $\delta u_{l, j}^{(n)}$, and the iterative dynamics of asynchronous control clusters results in updates that lead to decrease (for positive $\gamma$ ) or increase (for negative $\gamma$ ) of the metric $J$ value at every iteration 
step, assuming that the perturbations and $\gamma$ are suitably small in amplitude. ${ }^{9}$

\section{NUMERICAL SIMULATIONS}

\section{A. Adaptive Receiver with Zernike Stochastic Parallel Gradient Descent Clusters}

Consider an AO receiver system with wavefront control based on asynchronous SPGD clusters as shown in Fig. 1. Assume for simplicity that phase aberrations $\varphi(\mathbf{r}, t)$ originate from a thin phase-distorting layer (phase screen) located at the system's pupil plane, $z=0$. The complex amplitude of the input field after passing through the phase screen is $A_{\text {in }}(\mathbf{r}, t)=A_{0} \exp [i \varphi(\mathbf{r}, t)]$, where $A_{0}$ is the complex amplitude of a plane wave entering the phase screen. Propagation through the system optical train results in an additional (controllable) wavefront phase modulation $u(\mathbf{r}, t)$ introduced by a single or by multiple wavefront correctors. The system's output wave with complex amplitude $\quad A_{\text {out }}(\mathbf{r}, t)=A_{0} \exp [i \delta(\mathbf{r}, t)], \quad$ where $\quad \delta(\mathbf{r}, t)=\varphi(\mathbf{r}, t)$ $+u(\mathbf{r}, t)$ is the residual phase, enters the performance metric sensor (Strehl ratio sensor) composed of a lens with a small pinhole located in front of a photodetector at the lens's focal plane coordinate origin as shown in Fig. 1. The optical field complex amplitude in the lens focal plane is proportional to the two-dimensional Fourier transform of the field $A_{\text {out }}$ entering the lens. Thus the measured metric $J(t)$ is proportional to the output field intensity inside a small pinhole that, in its turn, is proportional to the output field zero-spectral component squared modulus

$$
J(t)=\left|\int A_{0} \exp [i \delta(\mathbf{r}, t)] \mathrm{d}^{2} \mathbf{r}\right|^{2}
$$

Assume that the phase modulation $u(\mathbf{r}, t)$ introduced by the wavefront corrector(s) can be represented as a sum of $N_{Z}$ classical aberrations described by the Zernike polynomials (Zernike aberrations) $\left\{Z_{k}(\mathbf{r})\right\}$

$$
u(\mathbf{r}, t)=\sum_{k=1}^{N_{z}} v_{k}(t) Z_{k}(\mathbf{r}),
$$

where $v_{k}(t)$ are the controlling amplitudes. We exclude in the sum [Eq. (9)] the zero-order Zernike polynomial $Z_{0}(\mathbf{r})=1$, corresponding to piston-type aberration, because the Strehl metric [Eq. (8)] does not depend on any constant phase shift.

Subdivide the control channels into $N_{c}$ SPGD clusters using the Zernike polynomial number (aberration order) as a distinguishing factor. The first cluster includes $M_{1}$ low-order Zernike aberrations (tip-tilt or defocus, etc.). The next (second) cluster incorporates control channels associated with compensation of the next $M_{2}$ Zernike aberrations, and so on. For convenience of counting the aberrations, we define response functions $S_{l, j}(\mathbf{r}) \equiv Z_{q}(\mathbf{r})$, where the polynomial index $q$ is coupled with the cluster number $j$ and control channel number $l$ inside the cluster by the following relationship: $q=l+\sum_{k=1}^{j-1} M_{k}$. The same counting rule is applied for both the aberration amplitudes $\mathbf{v}_{j}(t)=\left\{v_{l, j}(t)\right\}$ and controls $\mathbf{u}_{j}(t)=\left\{u_{l, j}(t)\right\}$.

Assume that the evolution of the amplitudes $\boldsymbol{v}_{j}(t)$ in response to applied controls $\boldsymbol{u}_{j}(t)$ is described by the system of equations [Eq. (2)] and that time responses $\left\{\tau_{j}\right\}$ are shorter for the clusters controlling higher-order aberrations. This sequence of time responses is associated with the corresponding sequence of characteristic times for the atmospheric turbulence induced phase aberration components. For these components the power spectrum bandwidth increases with the order of aberration. ${ }^{1}$ In the numerical simulations the sequence of time responses $\left\{\tau_{j}\right\}$ obeyed the following general scheme:

$$
\begin{array}{r}
\tau_{1}=\tau_{\max }, \quad \tau_{2}=\tau_{1}-\Delta, \quad \tau_{j}=\min \left\{\tau_{j}\right\} \\
+\left(N_{c}-j\right) \Delta \tau, \\
\text { for } j=2, \ldots, N_{c},
\end{array}
$$

where $\tau_{\max }=\max _{j}\left\{\tau_{j}\right\}, \Delta$ is a response time offset between the first cluster (the slowest one that corresponds to compensation of first $M_{1}$ Zernike aberrations) and the second cluster, $\tau_{N_{c}}=\min _{j}\left\{\tau_{j}\right\}=\tau_{\min }$ is the fastest control cluster

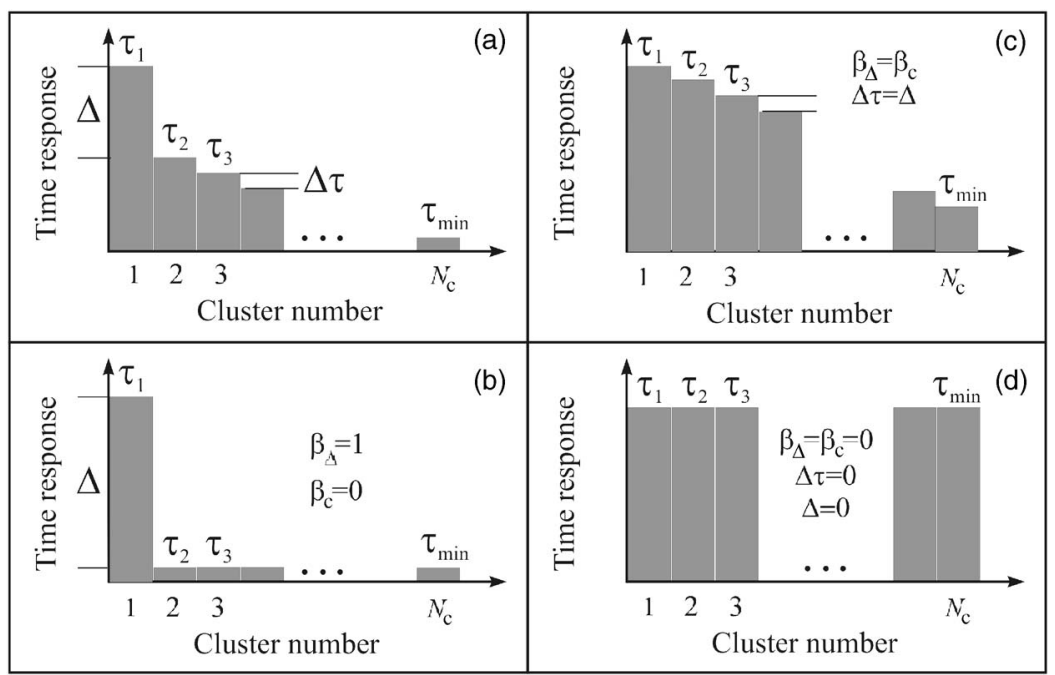

Fig. 3. Characteristic diagrams of time responses $\left\{\tau_{j}\right\}$ for adaptive system with $N_{c}$ asynchronous control clusters. (a) Definitions of time offsets $\Delta$ and $\Delta \tau$ in Eq. (10), (b)-(d) correspond to the numerical simulation results as described in the text. 
that corresponds to the highest-order aberrations compensation, and $\Delta \tau$ is the response time difference between the group of neighboring clusters excluding the first and the second clusters. The examples of the response time diagrams corresponding to Eq. (10) are shown in Fig. 3.

Define $\beta_{\tau}=\tau_{\max } / \tau_{\min }$ as the ratio between the slowest (first) and the fastest (last) clusters, and $\beta_{\Delta}=\Delta /\left(\tau_{\max }\right.$ $\left.-\tau_{\min }\right)$ as the normalized time offset between the first (slowest) and the second clusters. The initial time differences $\left\{\Delta_{j}\right\}$ between control voltage updates (initial intercluster time offset parameters) are chosen randomly inside the corresponding time intervals $\left\{\tau_{j}\right\}$. The feedback delay factor $\beta_{T}=T_{j} / \tau_{j}$ characterizing time delays $\left\{T_{j}\right\}$ between control voltage change and the corresponding metric measurements was set the same for all clusters.

Thus in our example the temporal characteristics of the network of SPGD clusters used in the numerical simulations is described by the following set of parameters: $\beta_{T}$ $=T_{j} / \tau_{j}, \quad \beta_{\Delta}=\Delta /\left(\tau_{\max }-\tau_{\min }\right), \quad \beta_{\tau}=\tau_{\max } / \tau_{\min }, \quad$ and $\quad \beta_{c}$ $=\Delta \tau /\left(\tau_{\max }-\tau_{\min }\right)$ - the normalized response time offset between the neighboring clusters.

In the numerical simulations, the parameters $\beta_{\tau}$ and $\beta_{T}$ were fixed $\left(\beta_{\tau}=5\right.$ and $\left.\beta_{T}=2\right)$ while the parameters $\beta_{\Delta}$ and $\beta_{c}$ were varied. The following intercluster time response distributions, shown in Figs. 3(b)-3(d), are considered in the computations:

1. Time responses of all clusters except the first (slowest) cluster are equal [see Fig. 3(b)]. This case corresponds to $\beta_{\Delta}=1$ and $\beta_{c}=0$.

2 . Time responses gradually decrease from cluster to cluster [Fig. 3(c)]: $\Delta \tau=\left(\tau_{\max }-\tau_{\min }\right) /\left(N_{c}-1\right)$ and $\beta_{\Delta}=\beta_{c}$ $=1 /\left(N_{c}-1\right)$.

3. All clusters have the same time responses [Fig. 3(d)]: $\beta_{\Delta}=\beta_{c}=0$.

Consider first the numerical simulation results for an AO system composed of $N_{c}=7$ asynchronous Zernike SPGD clusters and $N_{Z}=32$ is the total number of Zernike polynomials the AO system intends to compensate. The first cluster performs compensation of only wavefront tiptilt aberrations $\left(M_{1}=2\right)$. Each other control cluster includes control of five sequential Zernike aberrations. The pupil plane phase aberration (phase screen)is considered a random function with a power spectrum corresponding to the Kolmogorov atmospheric turbulence model. ${ }^{14}$ The aberration strength is characterized by the ratio $D / r_{0}$, where $D$ is the receiver aperture diameter and $r_{0}$ is the Fried parameter for a plane wave. ${ }^{15}$ The asynchronous SPGD cluster network dynamics is described by the system of Eqs. (2)-(5) and (8). The computation of Strehl ratio metric evolution curves $\mathrm{St}(t)$ during the adaptation process (instantaneous adaptation curves) is performed for fixed phase screen realizations $\{\varphi(\mathbf{r})\}$ over the time $t$ $=500 \tau_{1}$. Efficiency of adaptive compensation is characterized by the atmospheric-average dependence $\langle\operatorname{St}(t)\rangle$, obtained by averaging the instantaneous curves $\mathrm{St}(t)$ obtained for $N_{\varphi}=50$ different phase screen realizations. For simplicity we assumed that the metric sensor integration time, $\tau_{J}$ in Eq. (4), is significantly smaller than the characteristic time $\tau_{\min }$ and can be neglected. The numerical simulation of input wave propagation through the adap-

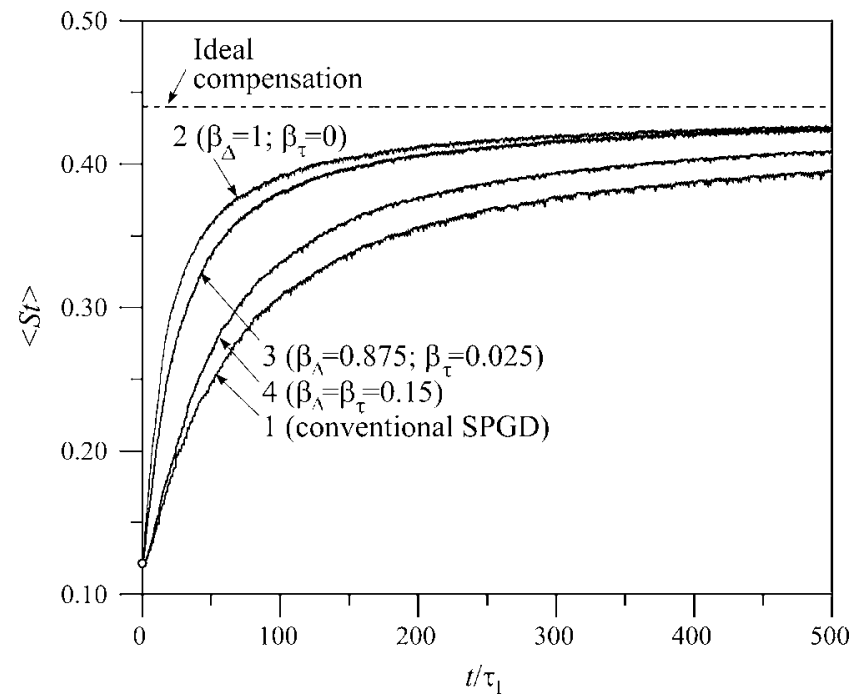

Fig. 4. Atmospheric-average Strehl metric evolution curves $\langle\operatorname{St}(t)\rangle$ obtained for the conventional SPGD system (curve 1), and for the control system with seven asynchronous SPGD clusters (curves 2-4). The cluster time response parameters correspond to the diagrams are shown in Fig. 3: (b) for curve 2, (c) for curve 4, and (d) for curve 1. The compensation level $\langle\mathrm{St}\rangle$ is defined as the ideal compensation is calculated using Strehl metric calculation based on residual phase aberrations $\delta(\mathbf{r})=\varphi(\mathbf{r})-\varphi_{Z}(\mathbf{r})$, where phase function $\varphi_{Z}(\mathbf{r})$ corresponds to the approximation of function $\varphi(\mathbf{r})$ with first $N_{Z}$ Zernike polynomials. Numerical simulations are performed for the Kolmogorov turbulence model with $D / r_{0}=4$.

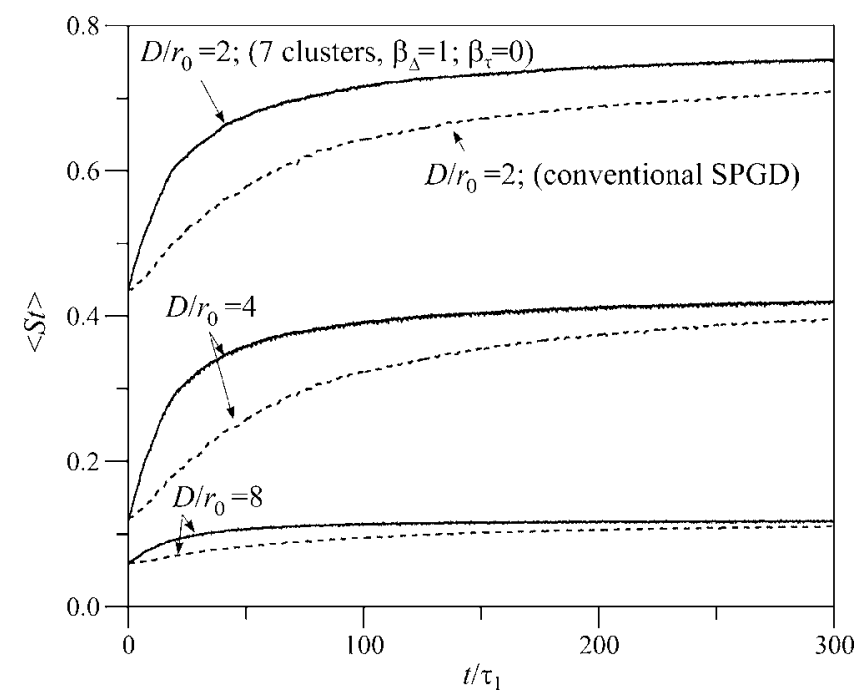

Fig. 5. Strehl ratio evolution curves $\langle\operatorname{St}(t)\rangle$ for SPGD (dashed lines) and the SPGD cluster (solid lines) AO system architectures for different atmospheric turbulence strengths defined by the $D / r_{0}$ ratio. The clusters' time responses correspond to diagram (b) in Fig. 3.

tive receiver system optical train was performed on a $256 \times 256$ numerical grid. The adaptation process evolution curves $\langle\mathrm{St}(t)\rangle$ are shown in Fig. 4 for the conventional SPGD control system corresponding to a single cluster with time response $\tau_{1}$ (curve 1), and for an AO system with seven asynchronous SPGD Zernike control clusters (curves 2-4) having different time response parameters $\beta_{\Delta}$ and $\beta_{c}$. In all cases, considered AO wavefront control based on asynchronous SPGD clusters demonstrated faster convergence that the conventional SPGD algorithm 
(compare curves $2-4$ with curve 1 ). The fastest convergence rate is achieved in the system with $\beta_{\Delta}=1$ and $\beta_{c}$ $=0$ shown in Fig. 4 by curve 2 . In this case, the clusters corresponding to high-order aberration compensation (all clusters except the first) have the fastest time response: $\tau_{j}=\tau_{\min }=0.2 \tau_{\max }$ for $j=2, \ldots, 7$ [see time-response diagram in Fig. 3(b)]. With the redistribution of time responses within the interval $\tau_{\min } \leqslant \tau_{j} \leqslant \tau_{\max }$ (by keeping the ratio $\beta_{\tau}=\tau_{\max } / \tau_{\min }=5$ unchanged) the adaptation rate drops as illustrated in Fig. 4 (compare curve 3 and 4 with curve 2) but still remains better than for the conventional SPGD (single cluster) control system architecture.

The advantage in the convergence rate achieved using the AO compensation with asynchronous SPGD clusters versus conventional control algorithm remains with variation of atmospheric turbulence strength as described by the $D / r_{0}$ ratio. The atmospheric average adaptation curves obtained for different $D / r_{0}$ values using the conventional and asynchronous SPGD control are shown in Fig. 5.

The adaptation curves in Figs. 4 and 5 demonstrate that despite the intercluster cross talk, the wavefront phase control using asynchronous SPGD clusters results in a stable monotonic convergence process. Contrary to the conventional SPGD approach for which the convergence rate is determined by the slowest control channel ("the slowest sets the pace" strategy), in AO systems based on parallel asynchronous SPGD clusters the convergence rate depends on characteristic response times of all clusters. The presence of "fast" clusters speeds up convergence for the entire system thus taking advantage of fast control loops.

\section{B. Adaptive Optical Receiver with Segmented and Tip-Tilt Mirrors}

Assume that the optical train of an adaptive receiver system in Fig. 1 includes the following two wavefront correctors: a tip-tilt (beam steering) mirror and a segmented mirror. The segmented mirror is composed of $N_{c}$ circular subapertures of diameter $d$ densely packed into a composite receiver aperture of diameter $D$. An example of a segmented mirror aperture comprising seven segments (subapertures) is shown in Fig. 1(b).

Assume that each subaperture has $N_{Z}$ actuators that can provide independent control of $N_{Z}$ low-order Zernike aberrations within the subaperture regions $\left\{\Omega_{j}\right\}$ defined by the expressions $\left\{\left|\mathbf{r}-\mathbf{r}_{j}\right| \leqslant d\right\}$, where $\left\{\mathbf{r}_{j}\right\}$ are the subaperture center points. The control voltages $\boldsymbol{u}_{j}(t)=\left\{u_{l, j}(t)\right\}($ $l=1, \ldots, N_{Z}$ and $\left.j=1, \ldots, N_{c}\right)$ applied to $j$ th subaperture, result in the phase modulation $u_{j}(\mathbf{r}, t)$ inside the corresponding subaperture region $\Omega_{j}$.

It is convenient to represent $u_{j}(\mathbf{r}, t)$ as the sum of the subaperture average phase $u_{j}^{p}(\mathbf{r}, t)$ (piston-type aberration), where $u_{j}^{p}(\mathbf{r}, t)=u_{j}^{p}(t)$ for $\left|\mathbf{r}-\mathbf{r}_{j}\right| \leqslant d$ and zero otherwise, and the aberration $u_{j}^{a}(\mathbf{r}, t)$ composed of the Zernike polynomials $\left\{Z_{l}(\mathbf{r})\right\}$ of higher than zero order:

$$
\begin{aligned}
u_{j}(\mathbf{r}, t) \equiv & u_{j}^{p}(\mathbf{r}, t)+u_{j}^{a}(\mathbf{r}, t)=v_{0, j}(t) Z_{0}\left(\mathbf{r}-\mathbf{r}_{j}\right) \\
& +\sum_{l=1}^{N_{z}} v_{l, j}(t) Z_{l}\left(\mathbf{r}-\mathbf{r}_{j}\right) .
\end{aligned}
$$

Here $\boldsymbol{\nu}_{j}(t)=\left\{\nu_{l, j}(t)\right\}$ are Zernike aberration amplitudes re- sulting from the applied control voltages $\left\{u_{l, j}(t)\right\}$. In Eq. (11) the Zernike polynomials $\left\{Z_{l}\left(\mathbf{r}-\mathbf{r}_{j}\right)\right\}$ are defined inside $\Omega_{j}$. Note that for the zero-order polynomial $Z_{0}\left(\mathbf{r}-\mathbf{r}_{j}\right)=1$. The amplitudes $\boldsymbol{\nu}_{0}(t)=\left\{\nu_{0, j}(t)\right\}$ are associated with the piston-type aberration and referred to here as the spatially uniform phase shifts (or just phase shifts).

The phase modulation $u_{\mathrm{SM}}(\mathbf{r}, t)$ introduced by the segmented mirror is given by the expression

$$
u_{\mathrm{SM}}(\mathbf{r}, t)=\sum_{j=1}^{N_{c}} u_{j}^{p}(\mathbf{r}, t)+\sum_{j=1}^{N_{c}} u_{j}^{a}(\mathbf{r}, t),
$$

for $\left|\mathbf{r}-\mathbf{r}_{j}\right| \leqslant d / 2\left(j=1, \ldots, N_{c}\right)$ and $u_{\mathrm{SM}}(\mathbf{r}, t)=0$ otherwise. We represent the phase modulation component introduced by the tip-tilt mirror in the form $u_{\mathrm{TM}}(\mathbf{r}, t)=\boldsymbol{\nu}_{T}(t) \mathbf{r}$, where $\mathbf{v}_{T}(t)=\left\{v_{x}(t), v_{y}(t)\right\}$ and $\mathbf{u}_{T}(t)=\left\{u_{x}(t), u_{y}(t)\right\}$ are the tip-tilt amplitude and control vectors, respectively. The complex amplitude of the optical wave that passes the receiver system optical train and enters the metric sensor (see Fig. 1) is given by

$$
\begin{aligned}
A_{\text {out }}(\mathbf{r}, t) & =A_{\text {in }} \exp [i \delta(\mathbf{r}, t)] \\
& =A_{\text {in }} \exp \left[i \varphi(\mathbf{r}, t)+i u_{\mathrm{SM}}(\mathbf{r}, t)+i u_{\mathrm{TM}}(\mathbf{r}, t)\right],
\end{aligned}
$$

where $\delta(\mathbf{r}, t)$ is the uncompensated (residual) phase aberration.

Assume that temporal dynamics of both the segmented and tip-tilt mirrors can be described by the system of equations, similar to Eq. (2), coupling the phase modulation amplitudes and controls:

$$
\begin{aligned}
\tau_{\mathrm{SM}} \frac{\mathrm{d} \mathbf{v}_{j}(t)}{\mathrm{d} t}+\mathbf{v}_{j}(t) & =\mathbf{u}_{j}(t) \quad j=1, \ldots, N_{c}, \\
\tau_{p} \frac{\mathrm{d} \mathbf{v}_{0}(t)}{\mathrm{d} t}+\mathbf{v}_{0}(t) & =\mathbf{u}_{0}(t), \\
\tau_{\mathrm{TM}} \frac{\mathrm{d} \mathbf{v}_{T}(t)}{\mathrm{d} t}+\mathbf{v}_{T}(t) & =\mathbf{u}_{T}(t),
\end{aligned}
$$

where $\mathbf{u}_{j}(t)=\left\{u_{l, j}(t)\right\}$ and $\mathbf{v}_{j}(t)=\left\{v_{l, j}(t)\right\}$ are vectors of the controls and phase modulation amplitudes for the $j$ th subaperture, $\tau_{\mathrm{SM}}$ is the time response of the segmented mirror control channels (excluding control of the piston-type aberration), and $\tau_{p}$ and $\tau_{\mathrm{TM}}$ are time responses of pistontype and tip-tilt aberrations, respectively.

Assign the AO system control channels to $N_{c}+2$ SPGD clusters operating in parallel. The first $N_{c}$ clusters, each composed of $N_{Z}$ control channels, control individual segmented mirror subapertures (except piston control). An additional control cluster is dedicated to control of only piston-type aberration. This cluster includes $N_{c}$ control channels. The last cluster combines the two control channels of the tip-tilt mirror.

In the numerical simulations, we considered a segmented mirror composed of $N_{c}=7$ closely located circular subapertures as shown in Fig. 1(b). The input-wave phase 


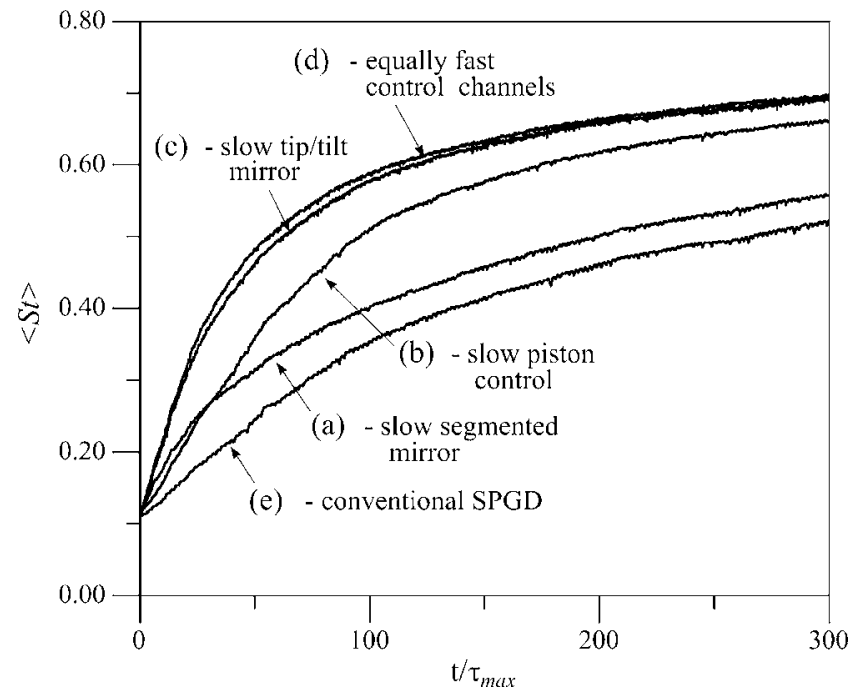

Fig. 6. Strehl ratio atmospheric-average adaptation curves $\langle\mathrm{St}(t)\rangle$ for the control system architecture shown in Fig. 1 with a segmented mirror (seven subapertures) and a tip-tilt mirror. A single metric (Strehl ratio) is used to control all channels. Control is based on asynchronous SPGD clusters for (a)-(d) and the conventional SPGD approach for (e). Numerical simulations are performed for $D / r_{0}=4, N_{Z}=32, \beta_{\tau}=\tau_{\max } / \tau_{\min }=4$ and $\beta_{T}=2$.

distortions are modeled using pupil plane phase screens corresponding to the Kolmogorov turbulence model. An example of a random phase screen is shown Fig. 1(a). The output signal of the Strehl ratio sensor, $J(t)=\mathrm{St}(t)$, defined by Eq. (8), was used as the input signal for all control clusters as illustrated in the optical system schematic in Fig. 1. For simplicity we assumed that the metric sensor's integration time, $\tau_{J}$ in Eq. (4), is significantly smaller than the characteristic times $\tau_{\mathrm{SM}}, \tau_{p}$, and $\tau_{\mathrm{TM}}$ and can be neglected.

The dynamics of the segmented and the tip-tilt mirrors were modeled using the discrete-time version of Eqs. (14)-(16). The computations of the control voltage update in the asynchronous SPGD clusters were performed using Eq. (5). The parameters $\beta_{\tau}=\tau_{\max } / \tau_{\min }$, and $\beta_{T}=T / \tau_{\max }$ (time delay factor) characterizing temporal characteristics of the control clusters were fixed $\left(\beta_{\tau}=4\right.$ and $\left.\beta_{T}=2\right)$ for all cases considered. Here $\tau_{\max }$ and $\tau_{\min }$ are maximum and minimum values of the response times $\tau_{\mathrm{SM}}, \tau_{p}$ and $\tau_{\mathrm{TM}}$.

The atmospheric-average performance metric evolution curves $\langle\operatorname{St}(t)\rangle$ are shown in Fig. 6 for the following cases: (a) $\tau_{\max }=\tau_{\mathrm{SM}}, \tau_{\min }=\tau_{\mathrm{TM}}=\tau_{p}$ (slow low-order aberrations control at the segmented mirror subapertures), (b) $\tau_{\max }$ $=\tau_{p}, \tau_{\min }=\tau_{\mathrm{SM}}=\tau_{\mathrm{TM}}$ (slow piston control), (c) $\tau_{\max }=\tau_{\mathrm{TM}}$, $\tau_{\min }=\tau_{\mathrm{SM}}=\tau_{p}$ (slow tip-tilt control), (d) $\tau_{\min }=\tau_{\mathrm{TM}}=\tau_{\mathrm{SM}}=\tau_{p}$ (equally fast control channels), (e) $\tau_{\max }=\tau_{\mathrm{TM}}=\tau_{\mathrm{SM}}=\tau_{p}$ (equally slow control channels, that corresponds to the conventional SPGD with $\beta_{\tau}=1$ and $\beta_{T}=2$ ). In the last case the slowest channels set the pace, so that all time responses were set to $\tau_{\max }$.

As seen from the comparison of the metric evolution curves in Fig. 6, subdivision of the AO system control channels into asynchronously operated SPGD clusters results in significant improvement of the adaptation convergence rate. The general tendency is that the convergence speed is dependent on the total number of the slow operating control channels.

\section{Distributed Stochastic Parallel Gradient Descent Control of Adaptive Receiver with Segmented and Tip-Tilt Mirrors}

The adaptation convergence rate can be significantly improved by combining the asynchronous SPGD cluster based wavefront control concept considered here with the distributed adaptive optics (DAO) concept. ${ }^{10,11}$ Assume that a single metric sensor is replaced by an array of the metric sensors that can provide an estimation of phase aberration compensation performance for the individual subapertures of the segmented mirror. An example of such an array of metric sensors is shown in Fig. 7. The sensor is composed of a lens array with a photo array located at its focal plane. The lenslets capture the output wavefront only inside regions corresponding to the segmented mirror subapertures $\left\{\Omega_{j}\right\}$. Thus, the subapertures of both the segmented mirror and the metric sensor (lenslet apertures) are geometrically matched. For simplicity, assume that both the aperture sizes and the center points $\left\{\mathbf{r}_{j}\right\}$ of the lenslets and subapertures coincide. Assume that the photo-array pixel size is smaller than the diffraction limited focal spot size corresponding to the segmented mirror subaperture size $d$. Efficiency of phase aberration compensation for the wavefront local regions $\left\{\Omega_{j}\right\}$ can be characterized using photocurrent measurements $\left\{J_{j}(t)\right\}\left(j=1, \ldots, N_{c}\right)$ obtained from a set of photo-array pixels located at the lenslet focal plane points $\left\{\mathbf{r}_{j}\right\}$. The signals $\left\{J_{j}(t)\right\}$, referred to here as the local metrics, are proportional to the Strehl ratios associated with the

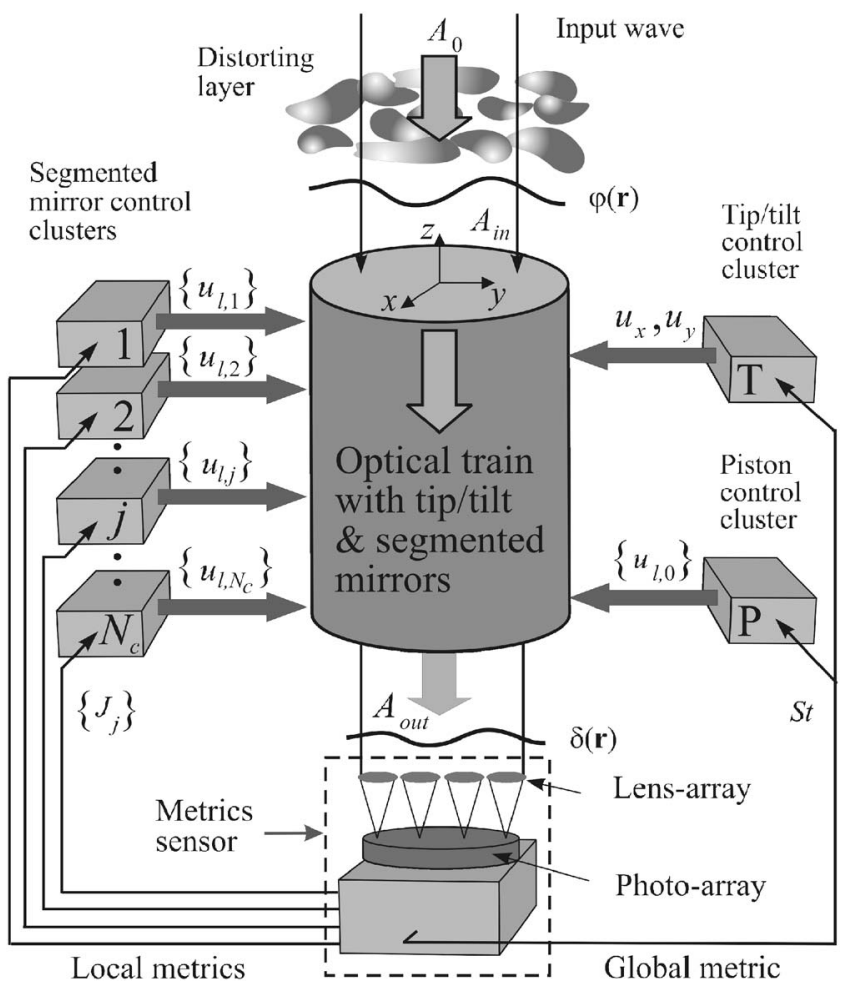

Fig. 7. Schematic of the AO receiver system with distributed control based on asynchronous SPGD clusters. The system includes a segmented mirror with seven subapertures and a tiptilt mirror. For both the piston and tip-tilt control clusters the global metric $J$ is used. The local metrics $\left\{J_{j}\right\}$ that correspond to the segmented mirror subapertures are used for controlling of low-order aberrations at the segmented mirror subapertures. 


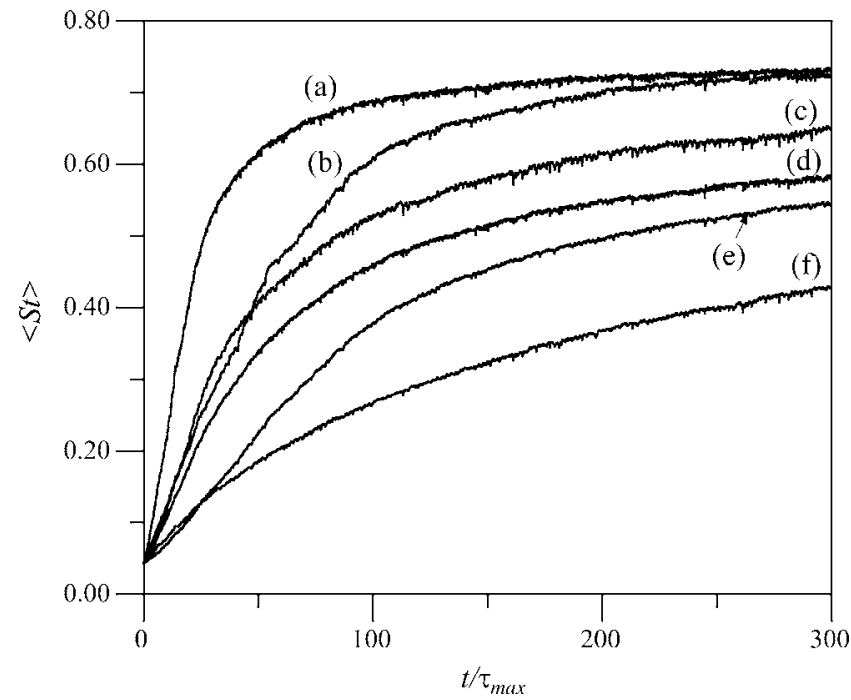

Fig. 8. Strehl ratio atmospheric-average adaptation curves for the DAO control systems architectures shown in Fig. 7 [curves (a)-(c)] and for the AO system architecture in Fig. 1 with asynchronous SPGD control using a simple metric [curves (d)-(f)]. In both cases control is based on asynchronous SPGD clusters with the following time responses: $\tau_{\max }=\tau_{\mathrm{SM}} \tau_{\min }=\tau_{\mathrm{TM}}=\tau_{p}$ (slow segmented mirror) for curves (c) and (f); $\tau_{\max }=\tau_{p}, \tau_{\min }=\tau_{\mathrm{SM}}=\tau_{\mathrm{TM}}$ (slow piston mirror) for (b) and (e); and $\tau_{\min }=\tau_{\mathrm{TM}}=\tau_{\mathrm{SM}}=\tau_{p}$ (equally fast control channels) for (a) and (d). Numerical simulations are performed for the Kolmogorov turbulence model with $D / r_{0}=8$ and $N_{Z}=32$.

segmented mirror subapertures. Note that the metric sensor in Fig. 7 is similar to Shack-Hartman sensors commonly used in AO systems for wavefront phase measurements. In the case considered, the Shack-Hartman-type sensor is used for measurements of the local metrics values but not to determine wavefront phase. As shown in Fig. 7, the local metrics $\left\{J_{j}(t)\right\}$ are used only by the SPGD control clusters performing compensation of Zernike aberrations at the segmented mirror subapertures. Note that the local metrics $\left\{J_{j}(t)\right\}$ cannot be used for compensation of the piston-type aberration as the constant phase shifts introduced by the piston control does not impact the local metric values. Compensation of the piston-type aberration can be performed using the Strehl ratio metric $J(t)$ $=\operatorname{St}(t)$, which is here referred to as the global metric. The metric $\operatorname{St}(t)$ is defined over the entire segmented mirror aperture area [see Eq. (8)]. The global metric $\operatorname{St}(t)$ can be measured using an additional metric sensor (not shown in Fig. 7) that shares the AO system output wavefront. Note that $\operatorname{St}(t)$ can be also calculated using the focal-plane intensity distribution measurements performed by the photo array. For the AO system schematic shown in Fig. 7, the global metric $\mathrm{St}(t)$ is also used for tip-tilt mirror control.

Results of the numerical simulation analysis of the $\mathrm{AO}$ system with DAO control described are presented in Fig. 8. In all cases considered, the use of distributed control resulted in significant improvement of the adaptive system performance-compare the atmospheric-average curves for DAO control [curves (a), (b), and (c)] with the corresponding evolution curves obtained using asynchronous SPGD optimization with a single metric [curves(d), (e), (f)] in Fig. 8.

\section{CONCLUSION}

In this paper we show that the problem of synchronization of multiple control loops of adaptive optics systems composed of several wavefront correctors with different time responses can be resolved without sacrificing performance of the entire adaptive system by setting the adaptation "clock" based on the slowest control channels. In the analyzed adaptive system architecture the control loops are combined into groups (clusters) that can be operated independently and asynchronously using the SPGD optimization algorithm. The physical reason why the asynchronously operating clusters do not interfere with each other in the sense that they preserve stability of the entire control system originates from the nature of the SPGD control. The asynchronous control voltage updates occurring in different SPGD clusters result in random variations of the system performance metric that are indistinguishable from the corresponding metric variations resulting from the control voltage perturbations normally used by SPGD control systems for the stochastic gradient estimation.

The results of the numerical simulations of two different adaptive receiver systems-one composed of a deformable mirror controlling Zernike aberrations and the second having both a tip-tilt mirror and a segmented wavefront corrector-show that the control system architectures based on asynchronously operated SPGD clusters can provide stable convergence of the optimization metric with a convergence rate exceeding that of a conventional AO system with an update rate based on the slowest wavefront control element present in the system.

The cluster-type structure of an adaptive control system also has an advantage from the hardware development viewpoint, as it offers scalable electronics control architectures. The SPGD clusters electronic control blocks can be easily combined in various configurations dependent on the specific requirements for wavefront distortion compensation without the need for reconfiguration of the entire control system. It is also shown that if the adaptation goal can be achieved by optimization of a set of metrics measured in parallel, asynchronous SPGD control clusters can provide simultaneous optimization of these metrics. This control system architecture, referred to as the distributed AO system (DAO), allows the system control channels to be partially decoupled and hence increase the adaptation process convergence rate. The analysis of the DAO system composed of an array of adaptive subapertures and the associated metric sensors controlled by the asynchronously operated clusters demonstrated significant adaptation performance improvement when compared with single-metric asynchronous SPGD cluster control.

Some AO applications, e.g., laser beam projection configurations using a relay mirror or laser communication systems, require remotely located adaptive systems to be operated in parallel. Wavefront control in these systems can be based on the cooperative sharing of the metric information. ${ }^{16}$ In these system types, wavefront control in both remotely located systems is performed using optimization of metrics measured locally and sent to other location via an optical or rf communication link. These systems can use asynchronous cluster SPGD control without 
the need for synchronization of clocks at both AO system locations. Recent experiments performed with free-space AO laser communication antennas using asynchronous control of an eight-channel deformable mirror at each end of the communication link provide experimental verification of the wavefront control technique introduced here. In these experiments the SPGD control clusters were used for optimization of the remotely measured metrics (received power). Metric exchange is performed through a full duplex rf communication channel. The results of these experiments will be presented in a subsequent paper.

\section{ACKNOWLEDGMENT}

This work was supported by the U.S. Joint Technology Office under contract JTO-02-602-18 and by the DARPA/ ATO project. The authors thank Konley Aschenbach for his help in the computer simulations.

M. A. Vorontsov can be reached by e-mail at Mvorontsov@arl.army.mil. His mailing address is 10766 W. Crestview Lane, Laurel, Maryland 20723. Phone, 301394-0214; fax, 301-394-0225.

\section{REFERENCES}

1. J. W. Hardy, Adaptive Optics for Astronomical Telescopes (Oxford U. Press, 1998).

2. D. C. Johnston and B. M. Welsh, "Analysis of multiconjugate adaptive optics,” J. Opt. Soc. Am. A 11, 394-408 (1994).

3. T. Weyrauch and M. A. Vorontsov, "Atmospheric compensation with a speckle beacon under strong scintillation conditions: directed energy and laser communication applications," Appl. Opt. 44, 1-14 (2005).

4. G. W. Carhart, M. A. Vorontsov, L. A. Beresnev, P. S. Paicopolis, and F. K. Beil, "Atmospheric laser communication system with wide angle tracking and adaptive compensation," in Free-Space Laser
Communication V, Proc. SPIE 5892, 589211-1-589211-12 (2005).

5. N. Nicolle, T. Fusco, G. Rousset, and V. Michau, "Improvement of Shack-Hartmann wavefront sensor measurement for extreme adaptive optics," Opt. Lett. 29, 2743-2745 (2004).

6. P. Madec, "Control techniques," in Adaptive Optics in Astronomy, F. Roddier, ed. (Cambridge U. Press, 1999), pp. 131-154.

7. R. A. Muller and A. Buffington, "Real-time correction of atmospherically degraded telescope images through image sharpening," J. Opt. Soc. Am. 64, 1200-1210 (1974).

8. M. A. Vorontsov, G. W. Carhart, and J. C. Ricklin, "Adaptive phase-distortion correction based on parallel gradient-descent optimization," Opt. Lett. 22, 907-909 (1997).

9. M. A. Vorontsov, G. W. Carhart, M. Cohen, and G. Cauwenberghs, "Adaptive optics based on analog parallel stochastic optimization: analysis and experimental demonstration," J. Opt. Soc. Am. A 17, 1440-1453 (2000).

10. M. A. Vorontsov, "Decoupled stochastic gradient descent optimization for adaptive optics: integrated approach for wave-front sensor information fusion," J. Opt. Soc. Am. A 19, 356-368 (2002).

11. S. L. Lachinova and M. A. Vorontsov, "Performance analysis of an adaptive phase-locked tiled fiber array in atmospheric turbulence conditions," in Target In the Loop: Atmospheric Tracking, Imaging and Compensation, Proc. SPIE 5895, 58950O-1-58950O-09 (2005).

12. M. A. Vorontsov and V. P. Sivokon, "Stochastic parallelgradient-descent technique for high-resolution wave-front phase-distortion correction," J. Opt. Soc. Am. A 15, 2745-2758 (1998).

13. J. C. Spall, Introduction to Stochastic Search and Optimization (Wiley, 2003).

14. A. N. Kolmogorov, "The local structure of turbulence in incompressible viscous fluids for very large Reynolds numbers," Dokl. Akad. Nauk SSSR 30, 301ff (1941).

15. D. L. Fried, "Optical resolution through a randomly inhomogeneous medium for very long and very short exposures," J. Opt. Soc. Am. 56, 1372-1379 (1966).

16. T. Weyrauch and M. A. Vorontsov, "Free-space laser communications with adaptive optics: atmospheric compensation experiments," in J. Opt. Fiber. Commun. Rep. 1, 355-379 (2004). 